\title{
Time trends of infant food allergy in China: three cross-sectional surveys in 1999,2009, and 2019
}

\author{
Zhuoying $\mathrm{Ma}^{1}$, Chen $\mathrm{Lin}^{2}$, Chen Jing ${ }^{3}$, Xian Ruoling ${ }^{4}$, Fang Heping ${ }^{4}$, Haiqi $\mathrm{Li}^{5}$, Wang \\ $\mathrm{Juan}^{6}$, and Yan $\mathrm{Hu}^{7}$ \\ ${ }^{1}$ Chongqing Medical University Affiliated Children's Hospital \\ ${ }^{2}$.Ministry of Education Key Laboratory of Child Development and Disorders, National \\ Clinical Research Center for Child Health and Disorders (Chongqing), China \\ ${ }^{3}$ Chongqing Youyoubaobei Women's and Children's Hospital. \\ ${ }^{4}$ Ministry of Education Key Laboratory of Child Development and Disorders, National \\ Clinical Research Center for Child Health and Disorders (Chongqing), China \\ ${ }^{5}$ Children's Hospital, Chongqing Medical University \\ ${ }^{6}$ Department of Child Health Care,Children's Hospital of Chongqing Medical University, \\ Chongqing, P.R. China. \\ ${ }^{7}$ Children's Hospital, Chongqing Medical University, Chongqing
}

July 23, 2020

\begin{abstract}
Background: Some studies have found a slowly increasing or decreasing trend of FA. However, the trends of FA in China are unknown. To investigate the prevalence of and characteristic changes in FA in children aged $<2$ years across 20 years. Methods: Data from three cross-sectional surveys on FA epidemiology conducted in 1999, 2009, and 2019 were assessed, and the outcomes were compared. The skin prick test was performed in all participants; open food challenge tests were used for diagnosis confirmation. Results: The prevalences of FA in 2009 and 2019 were significantly higher than that in 1999 (3.5\% in $1999,7.7 \%$ in 2009, $11.1 \%$ in 2019, $\mathrm{P}<0.001)$. However, there was no significant difference in prevalence between 2009 and 2019 $(\mathrm{P}=0.086)$. Cow's milk protein allergy $(5.7 \%)$ replaced egg allergy $(5.5 \%)$ as the most common food allergy in infants as per the results of the 2019 survey. The incidence of skin symptoms did not differ significantly over the study period (90.9\% in 1999 , $87.1 \%$ in 2009 , and $57.9 \%$ in $2019, \mathrm{P}>0.05$ ), while the incidence of gastrointestinal symptoms increased significantly over time ( $18.2 \%$ in $1999,12.9 \%$ in 2009 , and $43.9 \%$ in $2019, \mathrm{P}<0.05)$. Conclusion: The prevalence of FA in infants rapidly increased after the 1990s and gradually stabilized after 2010. Cow's milk protein has become the most common food allergen among infants in Chongqing, China. The incidence of gastrointestinal symptoms as a manifestation of FA has increased significantly since 2010.
\end{abstract}

\section{Hosted file}

Complete Manuscripts with Figures and Tables.doc available at https://authorea.com/ users/345477/articles/471699-time-trends-of-infant-food-allergy-in-china-three-crosssectional-surveys-in-1999-2009-and-2019 